\title{
Screening for cognitive decline following single known stroke using the Mini-Mental State Examination
}

This article was published in the following Dove Press journal:

Neuropsychiatric Disease and Treatment

12 April 201 I

Number of times this article has been viewed

\author{
David B Arciniegas 1,2,3 \\ Gregory F Kellermeyer ${ }^{1,2}$ \\ Nancy M Bonifer' \\ Kristin M Anderson-Salvi I \\ C Alan Anderson $2,3,4$ \\ 'Brain Injury Rehabilitation \\ Unit, HealthONE Spalding \\ Rehabilitation Hospital, Aurora, \\ CO, USA; ${ }^{2}$ Neuropsychiatry Service, \\ Department of Psychiatry, University \\ of Colorado Health Sciences Center, \\ Denver, CO, USA; ${ }^{3}$ Behavioral \\ Neurology Section, Department of \\ Neurology, University of Colorado \\ Health Sciences Center, Denver, CO, \\ USA; ${ }^{4}$ Neurology Service, Denver \\ Veterans Affairs Medical Center, \\ Denver, CO, USA
}

Correspondence: David B Arciniegas UCD Neurobehavioral Disorders Program, I300I East 17th Place,

Aurora, CO 80045, USA

$\mathrm{Tel}+\mathrm{I} 3037244998$

Fax +I 3037243594

Email david.arciniegas@ucdenver.edu
Background: Progressive cognitive decline develops in a nontrivial minority of stroke survivors. Although commonly used to identify cognitive decline in older stroke survivors, the usefulness of the Mini-Mental State Examination (MMSE) as a screening tool for post-stroke cognitive decline across a wider range of ages is not well established. This study therefore investigated the usefulness of the MMSE for this purpose.

Methods: Twenty-seven subjects, aged 18-82 years, with a single known remote stroke were assessed using the MMSE. The frequency of cognitive impairment was determined by comparison of MMSE scores with population-based norms. Relationships between cognitive performance, motor impairments, age, gender, handedness, stroke laterality, and time since stroke also were explored.

Results: Age-adjusted MMSE scores identified mild cognitive impairment in $22.2 \%$ and moderate-to-severe cognitive impairment in $7.4 \%$ of subjects. Raw and age-adjusted MMSE scores were inversely correlated with time since stroke, but not with other patient or stroke characteristics.

Conclusion: A relationship between time since single known stroke and MMSE performance was observed in this study. The proportion of subjects identified as cognitively impaired in this group by Z-transformation of MMSE scores using previously published normative data for this measure comports well with the rates of late post-stroke cognitive impairment reported by other investigators. These findings suggest that the MMSE, when normatively interpreted, may identify cognitive decline in the late period following single known stroke. Additionally, the lack of a relationship between MMSE and Fugl-Meyer scores suggests that the severity of post-stroke motor impairments is unlikely to serve as a clinically useful indicator of the need for cognitive assessment. A larger study of stroke survivors is needed to inform more fully on the usefulness of normatively interpreted MMSE scores as a method of screening for post-stroke cognitive decline.

Keywords: stroke, Mini-Mental State Examination, cognitive decline, Fugl-Meyer evaluation, motor impairment

\section{Introduction}

Improvement of acute stroke-induced cognitive impairments is expected over the months to years following stroke, ${ }^{1-8}$ with as many as $30 \%$ of stroke survivors experiencing complete cognitive recovery by 18 months post-stroke. ${ }^{9}$ Among persons who do not experience a complete recovery from post-stroke cognitive impairments, conventional clinical wisdom suggests that those individuals maintain persistent but stable cognitive impairments thereafter. However, a nontrivial minority of stroke 
survivors develop progressive cognitive decline over the first two years following a single known stroke.

For example, Ballard et $\mathrm{al}^{6}$ performed cognitive assessments three and 15 months following stroke in 115 individuals without overt dementia in the immediate post-stroke period. Although $50 \%$ of these subjects demonstrated cognitive improvements by 15 months following stroke, $9 \%$ declined cognitively over that same time period. These subjects were without prior or subsequent known strokes, suggesting that even a single known stroke may provoke vascular dementia. Other studies offer similar evidence of cognitive decline in the months to years following stroke, with rates of dementia by two years post-stroke of $9 \%-31 \% .^{8-11}$ In these studies, extended periods of observation after stroke (1-2 years) revealed higher rates of cognitive impairment than did studies with relatively short post-stroke observation periods (less than one year).

Other patient or stroke characteristics may facilitate the identification of persons at risk for post-stroke cognitive decline. Advanced age appears to be a risk factor for dementia following stroke, ${ }^{8}$ with a one-year post-stroke prevalence of dementia of $7 \%$ in those aged $<65$ years and $53 \%$ in those aged $>85$ years. ${ }^{12}$ Multivariate analyses of large stroke cohorts demonstrate associations between long-term poststroke cognitive impairment and educational level, ${ }^{13}$ lower socioeconomic status, ${ }^{14}$ ethnicity (Afro-Caribbean, Asian), ${ }^{14}$ stroke severity, ${ }^{13}$ left hemispheric lesion, ${ }^{14}$ prior cerebrovascular disease, ${ }^{13}$ dysphasia, ${ }^{13}$ visual field defect, ${ }^{14}$ and urinary incontinence. ${ }^{14}$ These studies suggest that some patient and/ or stroke characteristics, as well as medical comorbidities (eg, prior cerebrovascular disease, incontinence), may serve to prompt clinicians to evaluate patients with such characteristics for post-stroke cognitive decline.

From a practical standpoint, particularly in the busy clinical practices of neurologists, physiatrists, and primary care physicians caring for stroke survivors, screening for poststroke cognitive decline presents several challenges. First, in a time-limited setting, it is often impractical to administer more than a brief measure of general cognition, such as the Mini-Mental State Examination (MMSE). ${ }^{15}$ Formal neuropsychological testing is often useful for the identification and quantification of post-stroke cognitive impairments, but obtaining support for such testing is inconsistently available, especially in many managed care environments. ${ }^{16}$ As such, the task of assessing post-stroke cognitive performance is often relegated to primary care physicians, neurologists, psychiatrists, and physiatrists, and therefore the officebased assessment of cognition is frequently limited to the MMSE.
While the MMSE is not a substitute for formal neuropsychological testing, it appears to be a useful measure for the assessment of post-stroke cognitive decline. For example, Laukka et al ${ }^{17}$ suggest that the MMSE may be a useful measure with which to identify forthcoming vascular dementia in adults $\geq 75$ years of age, and Madureira et $\mathrm{al}^{18}$ found the MMSE to be a useful screening measure of cognition among older persons in the post-acute (three-month) period following stroke. However, the usefulness of the MMSE measure for the identification of post-stroke cognitive impairment across a broader age range and in the late (ie, more than one year) period following stroke has not been established.

Additionally, the types of stroke-related impairments associated with incipient post-stroke dementia noted above (eg, dysphasia, visual field defect, severity of initial stroke, urinary incontinence) are often challenging to identify and quantify in a brief office visit, particularly in non-neurological clinical settings. When such are identified, clinicians may be more likely to perform cognitive screening tests, assuming that the presence and severity of other stroke sequelae may serve as a gauge of the likelihood and/or severity of post-stroke cognitive impairments. However, it is possible that the relationship between cognitive and other stroke-related impairments may be an artifact of age, with older persons experiencing more frequent impairments in a variety of neurological and functional domains, regardless of whether there are causal relationships between such impairments. Accordingly, it would be useful to understand more fully the relationship between post-stroke motor and cognitive impairments in the late period following stroke, and particularly whether the former serve as a proxy with which to identify stroke survivors in need of more detailed cognitive assessment.

The present study was undertaken to address these issues by investigating the usefulness of the MMSE as a screening tool for post-stroke cognitive decline among younger stroke survivors, and particularly the utility of interpreting MMSE performance according to population-based norms for this purpose. Additionally, relationships between cognitive performance, motor performance, time since stroke, and a limited set of easily identified patient and stroke characteristics were investigated for the purpose of determining whether these variables serve usefully to identify survivors of remote strokes in need of cognitive assessment.

\section{Materials and methods}

This study was approved by the HealthONE Alliance Institutional Review Board, and all subjects provided informed consent for study participation. 


\section{Subjects}

Individuals who experienced a single known stroke at least 12 months prior to study participation were recruited nationally via printed and Internet media for participation in a study examining the effects of constraint-induced movement therapy on chronic post-stroke upper extremity motor impairments. Participants were enrolled on the basis of the onset and persistence of moderate-to-severe upper extremity motor impairments following a single known stroke, with moderateto-severe upper extremity motor impairment, defined as movement from a resting position limited to wrist extension of no more than $20^{\circ}$, metacarpophalangeal and interphalangeal joint extension of no more than $20^{\circ}$, but preserved ability to grasp a washcloth using any method of prehension. Subjects were also required to have the ability to sit at the bedside for 10 minutes without support, to follow directions using written, verbal, or demonstration instructions, and to have no other serious and/or uncontrolled medical conditions. Findings from the constraint-induced movement therapy protocol into which these subjects subsequently entered are described elsewhere. ${ }^{19-21}$ Medical records were reviewed for the purpose of determining stroke type and laterality.

\section{Outcome measures}

Subjects completed pretreatment assessments using the MMSE $^{15}$ and the Fugl-Meyer evaluation of physical performance. ${ }^{22}$ The MMSE is a brief cognitive assessment measure used commonly by physicians and allied health care providers in clinical practice. MMSE scores range between 0 and 30 , with higher scores reflecting better performance. This measure was administered and scored using the method described by Folstein et al. ${ }^{15}$ In order to account for the effect of age prior to interpreting MMSE scores, adjusted MMSE scores were calculated using the population-based norms reported by Crum et al. ${ }^{23}$ Mild cognitive impairment was defined as an MMSE score $\geq 1$ standard deviation (SD) below ageadjusted performance expectations, ${ }^{24}$ and moderate or greater cognitive impairment was defined as an MMSE score $\geq 2 \mathrm{SD}$ below age-adjusted performance expectations.

The Fugl-Meyer assessment generated a score for upper extremity performance (FM-UE) based on motor skill, coordination, and speed of upper extremity movement; FM-UE scores range from 0 to 66 , with lower scores reflecting more severe impairment. The Fugl-Meyer assessment also generates a total motor performance score (FM-T) based on the FM-UE and also joint range of motion, pain, and sensory function, as well as lower extremity function. For the purpose of this study, FM-T scores ranged from 0 to 126 points, again with lower scores reflecting more severe impairment. All administrations of the Fugl-Meyer assessment were completed by one occupational therapist following Fugl-Meyer testing guideline ${ }^{22}$ and employed a standardized assessment environment (ie, the same chair, testing equipment, and testing procedures used for every subject). Determination of handedness was also made during the course of Fugl-Meyer assessment. Test-retest reliability on both the FM-UE and FM-T were determined by repeat assessment of 10 randomly selected patients; for both measures, the Pearson product moment correlation was $\mathrm{r}=0.96(P<0.05)$.

\section{Statistical analyses}

All statistical analyses were performed using Statistica 6.0 (Statsoft Inc, Tulsa, OK). Pearson product moment correlation coefficients were calculated for age versus MMSE (raw and age-adjusted), age versus Fugl-Meyer (FM-UE or FM-T), time since stroke versus MMSE (raw and age-adjusted), time since stroke versus Fugl-Meyer (FM-UE or FM-T), and MMSE (raw and age-adjusted) versus Fugl-Meyer (FM-UE or FM-T). Student $t$-tests were used to investigate differences in MMSE and FM-T scores as a function of gender, laterality of stroke, and cerebral dominance. These analyses were cross-validated by dividing the study group into those with and without cognitive impairment (ie, age-adjusted performance $\geq 1$ SD below norm-based expectations) and then using Student $t$-tests to investigate between-group differences in age, time since stroke, FM-UE, and FM-T. $\chi^{2}$ analyses were used to investigate differences in gender, cerebral dominance, and laterality of stroke among subjects with and without cognitive impairment.

\section{Results}

Twenty-seven subjects (10 of whom were female) were included. The study group is described in Table 1 (continuous variables of interest) and Table 2 (categorical variables of interest). Mild cognitive impairment was observed in $6 / 27$ subjects $(22.2 \%)$, and moderate or greater cognitive

Table I Study group characteristics (continuous variables)

\begin{tabular}{llll}
\hline & Mean $( \pm$ SD) & Median & Range \\
\hline Age (years) & $58.5 \pm 16.8$ & 60.0 & $18-82$ \\
Time post-stroke (years) & $5.9 \pm 5.2$ & 3.5 & $1-20$ \\
MMSE & $27.3 \pm 3.4$ & 28.0 & $14-30$ \\
Age-adjusted MMSE & $-0.10 \pm 1.6$ & 0.4 & $-5.7-1.9$ \\
Fugl-Meyer (upper extremity) & $30.3 \pm 9.6$ & 26.0 & $17-51$ \\
Fugl-Meyer (total) & $84.8 \pm 11.8$ & 84.0 & $66-112$ \\
\hline
\end{tabular}

Abbreviations: SD, standard deviation; MMSE, Mini-Mental State Examination. 
Table 2 Study group characteristics (categorical variables)

\begin{tabular}{llr}
\hline Gender & 17 men & $63 \%$ \\
Handedness & 10 women & $37 \%$ \\
& 23 right & $85 \%$ \\
Hemispheric laterality of stroke & 3 left & $11 \%$ \\
& 1 mixed & $4 \%$ \\
Stroke type & 15 left & $56 \%$ \\
& 12 right & $44 \%$ \\
& 17 ischemic & $63 \%$ \\
& 6 hemorrhagic & $22 \%$ \\
& 1 mixed & $4 \%$ \\
& 3 undetermined & $11 \%$ \\
\hline
\end{tabular}

impairment was observed in $2 / 27$ subjects (7.4\%). Time since stroke was inversely correlated with both raw and age-adjusted MMSE scores $(r=-0.65, P<0.001$, and $r=-0.59, P<0.002$, respectively), but not with FM-UE or FM-T scores. Age was not correlated with raw or ageadjusted MMSE scores, but age was inversely correlated with FM-UE and FM-T scores (both $r=-0.47, P<0.02$ ). Neither raw nor age-adjusted MMSE scores correlated with FM-UE or FM-T scores. Relationships between ageadjusted MMSE scores, FM-T scores, and age are presented in Figure 1, and relationships between age-adjusted MMSE scores, FM-T scores, and time since stroke are presented in Figure 2. Raw and age-adjusted MMSE scores did not differ as a function of gender, cerebral dominance, or laterality of stroke. Similarly, FM-UE or FM-T scores did not differ as a function of gender, cerebral dominance, or laterality of stroke.

After dividing subjects into groups with and without cognitive impairment, there were no significant differences between these groups with respect to age, gender, cerebral dominance, laterality of stroke, FM-UE, or FM-T scores. However, time since injury was significantly longer among subjects with MMSE-determined cognitive impairment (10.3 \pm 8.4 years) when compared with subjects performing within normal limits for age on this measure ( $4.6 \pm 3.2$ years, $t=2.6, P<0.02)$.

\section{Discussion}

The present findings suggest that the MMSE, particularly when interpreted using age-adjusted normative data, may be useful in the identification of post-stroke cognitive impairment among both younger and older adult stroke survivors. This suggestion is consistent with the conclusions of other investigators ${ }^{25-27}$ and the American Heart Association. ${ }^{28}$

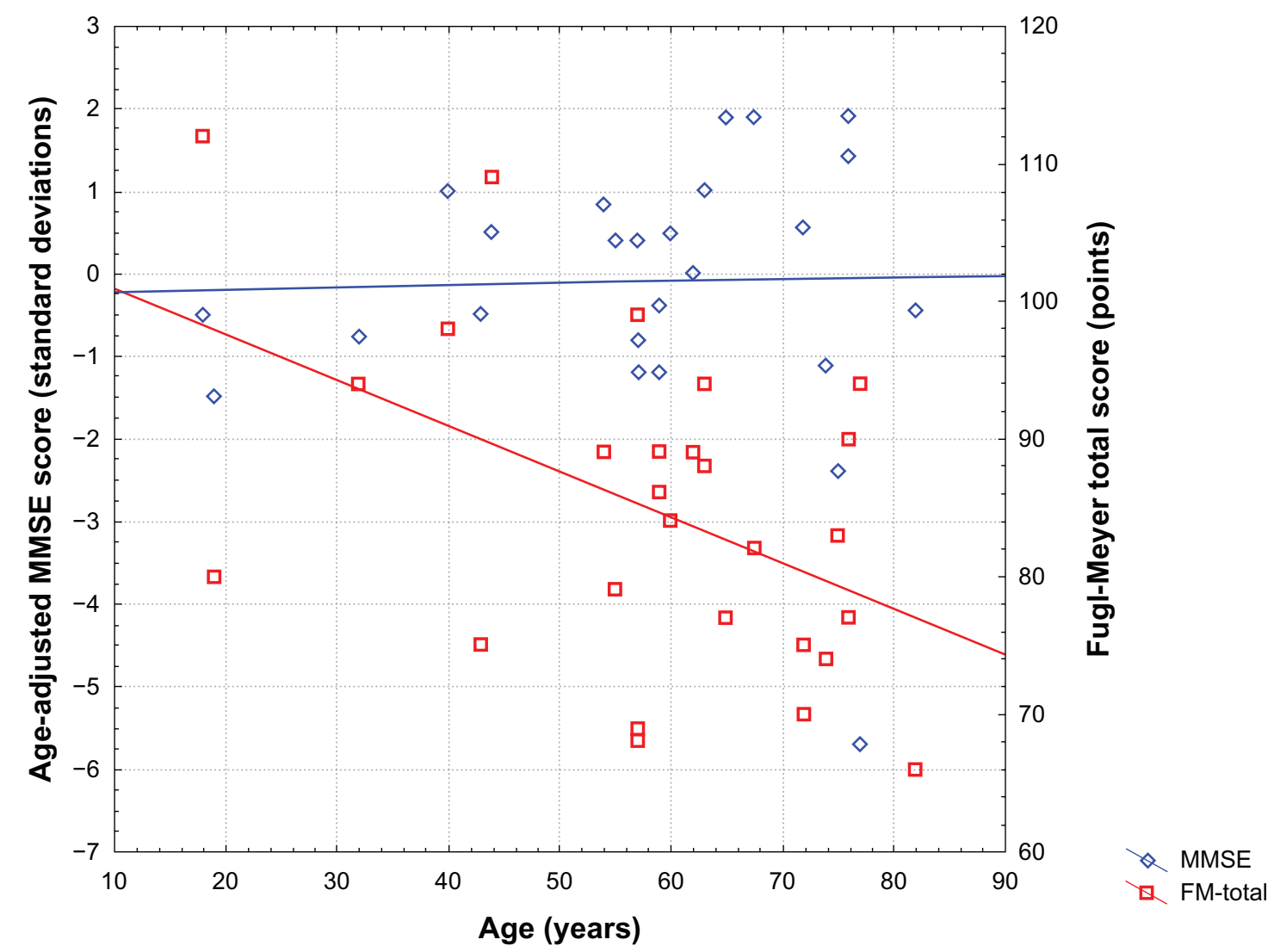

Figure I Relationships between age-adjusted Mini-Mental State Examination (MMSE) scores and age, and Fugl-Meyer total (FM-Total) scores and age. 


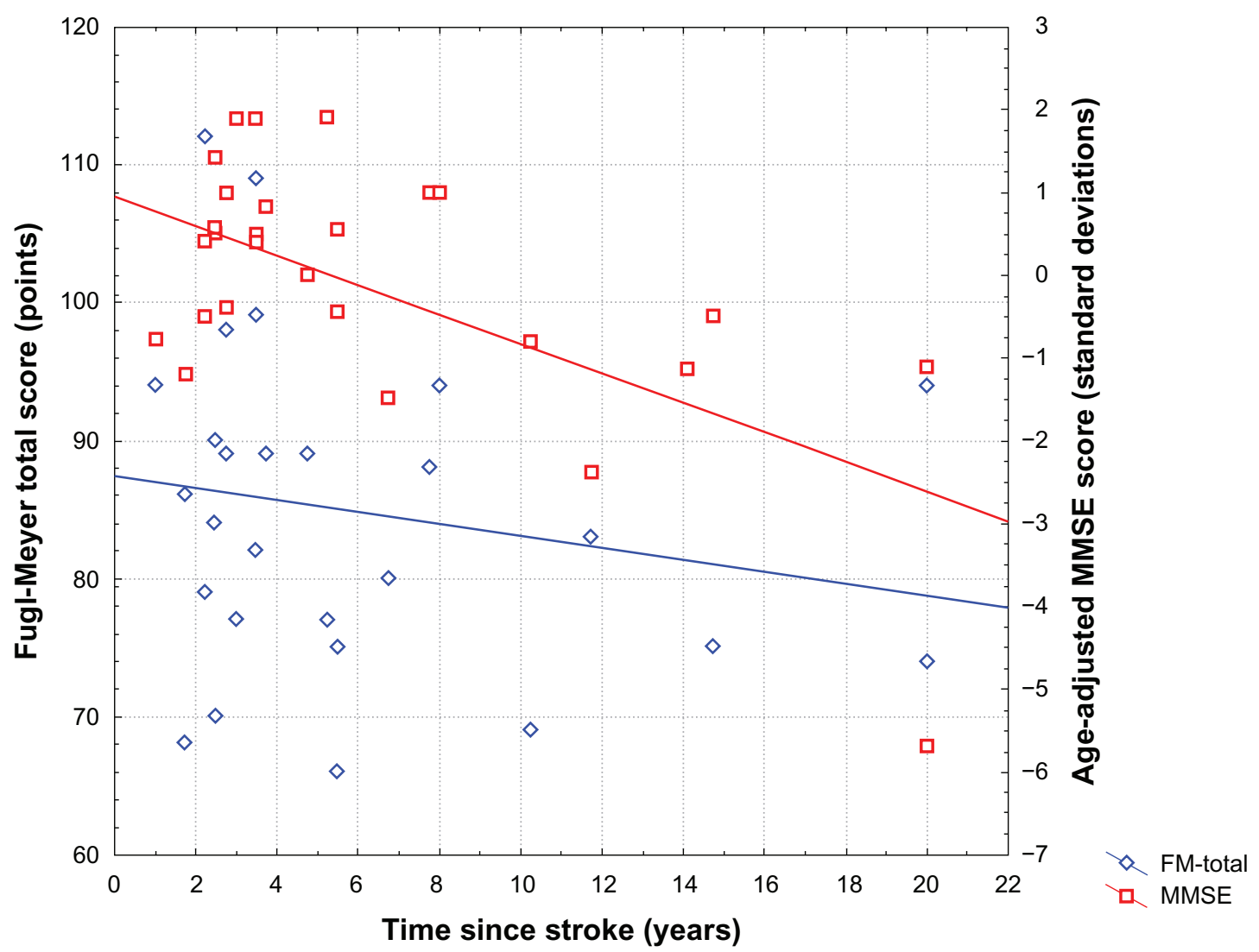

Figure 2 Relationship between age-adjusted Mini-Mental State Examination (MMSE) scores and time since stroke, and between Fugl-Meyer total (FM-Total) scores and time since stroke.

Our findings clarify these suggestions by demonstrating that the usefulness of the MMSE for this purpose relies upon Z-transforming scores on this measure. Age influences MMSE performance, and the magnitude of the effect of age on MMSE performance increases with advancing age. Accordingly, interpreting MMSE scores in a manner that adequately controls for the potential confound of agerelated performance decrements necessitates Z-transforming raw MMSE scores using the best available normative data. ${ }^{23}$ In this study, age-adjusted MMSE scores identified 22.2\% of subjects in this study with cognitive impairment of at least mild severity, $50 \%$ of whom were $\leq 60$ years of age (see Figure 1). Moderate or greater cognitive impairment (ie, vascular dementia) was identified in $7.4 \%$ of subjects, consistent with frequencies identified in studies using more extensive neuropsychological testing batteries. ${ }^{8-11}$ By comparison, using raw MMSE cutoff scores of $\leq 25$ or $\leq 24$ would identify only $18.5 \%$ or $7.4 \%$, respectively, of subjects in this group as cognitively impaired. The even more conservative cutoff score of $<20$ (for "organicity"), originally proposed by Folstein et al, ${ }^{15}$ would identify only $1 / 27$ $(3.7 \%)$ of subjects in this sample as cognitively impaired.
Therefore, we suggest that using raw MMSE score cutoff values to establish cognitive impairment is not appropriate, and may explain why some other groups conclude (perhaps erroneously) that this measure underestimates the frequency of post-stroke cognitive decline. ${ }^{29-32}$ Conversely, applying a less conservative raw MMSE cutoff score of $\leq 26$ to our sample overidentifies subjects (29.6\%) as having cognitive impairments of at least mild severity. Collectively, these observations suggest that the MMSE may be useful as an assessment for clinically significant post-stroke cognitive decline, and that the interpretation of MMSE scores for this purpose is best undertaken by comparing individual scores with published normative data. ${ }^{23}$

Cognitive performance as assessed by both raw and ageadjusted MMSE scores was inversely correlated with time since stroke, but was not correlated with the severity of poststroke motor impairments, age, gender, cerebral dominance, or laterality of stroke. By contrast, the severity of post-stroke motor impairment was correlated with age, but was not correlated with time since stroke or the other patient or stroke characteristics assessed in this study. The pattern of relationships between cognitive performance, motor function, age, 
and time since stroke observed in this study is complex. These relationships are considered individually and collectively in the service of considering their potential application to the care and future study of stroke survivors.

The correlation between cognitive performance and time since stroke suggests a time-related decline in cognition in the late period following stroke. Importantly, that decline is not accounted for by age, post-stroke motor impairment, or the other subject and stroke characteristics evaluated in this study. Although the association between increased severity of cognitive impairment and time since stroke observed in this study is likely to be multifactorial, two interpretations are immediately forthcoming.

First, it is possible that the cognitive performance of these subjects simply reflects their pre-stroke cognitive baseline, persistent and stable cognitive impairments since the time of stroke, or both, and that the apparent relationship between time since stroke and cognitive impairment is spurious. The strength of the association between time since stroke and both raw MMSE and age-adjusted MMSE scores suggests that the likelihood of a Type I error in this analysis is small, but this possibility cannot be dismissed entirely in light of the relatively small sample size of the present study.

Second, and more likely, our present findings suggest that a nontrivial minority of stroke survivors develop progressive cognitive decline in the late post-stroke period. That decline may result from the cumulative effects of additional (including otherwise clinically "silent") cerebrovascular disease, ${ }^{33-37}$ the induction of Alzheimer's-type neuropathology by cerebrovascular disease, ${ }^{38-41}$ or both of these and/ or other factors. ${ }^{42-46}$ This interpretation is concordant with findings from other similar studies, ${ }^{24,37,47-49}$ and suggests that a single known stroke is probably understood most usefully as an overt manifestation of an underlying cerebrovascular process that in a substantial minority of individuals will result in gradual cognitive decline.

In contrast with post-stroke cognitive performance, motor performance remained relatively more stable as a function of time since stroke. However, motor performance demonstrated a clear age-related decline. The quality of motor function varies with normal aging, ${ }^{50}$ and clinically apparent motor decline begins in the fifth decade of life. By contrast, the Crum et $\mathrm{al}^{23}$ data suggest that significant agerelated decline in MMSE scores is not expected until the eighth decade of life. These observations might suggest that age may more strongly influence motor performance than cognitive performance among relatively younger stroke survivors. Given that the mean age in the present study was
$58.5 \pm 16.8$ years, the present observation of a relationship between age and post-stroke motor performance, but not between age and MMSE scores, is not entirely unexpected.

It is also important to note that the severity of motor impairments experienced by the subjects in this study were just short of plegia of the affected limb or hemibody. The lack of correlation between post-stroke cognitive performance and motor performance is therefore even more important to highlight here. If in this group there is no significant association between motor and cognitive performance, then severity of motor impairments seems unlikely to serve usefully as an indicator of post-stroke cognitive impairments.

The present study suffers from several limitations, including its development as a secondary analysis of cognition in a sample of stroke survivors recruited for a different purpose (constraintinduced movement therapy of post-stroke motor impairments), cross-sectional rather than longitudinal assessment of cognition and motor function, nonblinded assessments, lack of a matched comparison sample, lack of extensive demographic data (eg, educational levels, ethnicity, primary language, socioeconomic status), absence of overall stroke severity metrics (eg, National Institutes of Health Stroke Scale ${ }^{51}$ ), lack of ascertainment of potential confounds such as neuropsychiatric conditions (ie, depression, anxiety, substance use) and neuroactive medications on cognitive and motor performance, and lack of assessment with the formal neuropsychological testing needed to establish the validity of the rates of cognitive impairment identified by Z-transformed MMSE scores. Of particular note, the recruitment strategy for the constraint-induced movement therapy study may at least in part contribute to the lack of correlation between motor and cognitive performance in the present sample. As noted earlier, subjects were required to be able to follow directions using written, verbal, or demonstration instructions. This requirement reduces the likelihood of enrolling subjects with functionally significant language impairments, and would tend to bias MMSE scores towards the less impaired range. Accordingly, these subjects were less likely than the general stroke population to demonstrate an association between motor and cognitive (including language) abilities. It is possible that, if subjects with more overt impairments of language had been included in the present study, a correlation between motor and cognitive performance might have been observed. Conversely, the finding of an association between time since stroke and cognitive performance despite the apparent selection bias against patients with aphasia is that much more noteworthy, because it suggests that post-stroke language disturbances alone are unlikely to explain the MMSE scores observed in these subjects. 
In summary, the present findings suggest that the MMSE may serve as a useful screening measure of post-stroke cognitive performance across a wide age range, particularly when MMSE scores are interpreted with respect to population-based norms rather than raw MMSE cutoff scores. Additionally, the present study findings suggest that clinicians should remain vigilant for the development of progressive cognitive decline throughout the post-stroke period, and that such vigilance should be maintained regardless of a patient's age and/or severity of post-stroke motor impairments. Given the morbidity and mortality risks posed by post-stroke cognitive impairment ${ }^{52-54}$ and promise of emerging therapies for the treatment of vascular dementia, ${ }^{55-61}$ routine screening for cognitive impairments among stroke survivors is necessary if such treatments are to be offered early in the course of vascular dementia, when preservation of function may yield the greatest benefits for affected persons and their families. The present findings suggest that identification of cognitive impairments rests upon direct assessment of cognition, and that recognition of other patient or stroke characteristics are neither suitable substitutes nor reliable prompts for poststroke cognitive assessment. Prospective studies are needed to validate the present findings, including direct comparison of the rates of cognitive impairment identified by Z-transformed MMSE scores versus formal neuropsychological testing, and to investigate further their clinical implications.

\section{Acknowledgments}

The authors gratefully acknowledge the support provided for this study by the Spalding Community Foundation, the HealthONE Alliance, and HealthONE Spalding Rehabilitation Hospital, Aurora, CO.

\section{Disclosure}

The authors report no conflicts of interest in this work.

\section{References}

1. Wade DT, Skilbeck C, Hewer RL. Selected cognitive losses after stroke. Frequency, recovery and prognostic importance. Int Disabil Stud. 1989;11(1):34-39.

2. Kinsella G, Ford B. Acute recovery from patterns in stroke patients: Neuropsychological factors. Med J Aust. 1980;2(12):663-666.

3. Enderby P, Wood VA, Wade DT, Hewer RL. Aphasia after stroke: A detailed study of recovery in the first 3 months. Int Rehabil Med. 1987;8(4):162-165.

4. Wade DT, Wood VA, Hewer RL. Recovery of cognitive function soon after stroke: A study of visual neglect, attention span and verbal recall. J Neurol Neurosurg Psychiatry. 1988;51(1):10-13.

5. Desmond DW, Moroney JT, Sano M, Stern Y. Recovery of cognitive function after stroke. Stroke. 1996;27(10):1798-1803.

6. Ballard C, Rowan E, Stephens S, Kalaria R, Kenny RA. Prospective follow-up study between 3 and 15 months after stroke: Improvements and decline in cognitive function among dementia-free stroke survivors $>75$ years of age. Stroke. 2003;34(10):2440-2444.
7. Hochstenbach JB, den Otter R, Mulder TW. Cognitive recovery after stroke: A 2-year follow-up. Arch Phys Med Rehabil. 2003; 84(10):1499-1504.

8. Patel M, Coshall C, Rudd AG, Wolfe CD. Natural history of cognitive impairment after stroke and factors associated with its recovery. Clin Rehabil. 2003;17(2):158-166.

9. Tham W, Auchus AP, Thong M, et al. Progression of cognitive impairment after stroke: One year results from a longitudinal study of Singaporean stroke patients. J Neurol Sci. 2002;203-204:49-52.

10. Kase CS, Wolf PA, Kelly-Hayes M, Kannel WB, Beiser A, D'Agostino RB. Intellectual decline after stroke: The Framingham Study. Stroke. 1998;29(4):805-812.

11. Prencipe M, Ferretti C, Casini AR, Santini M, Giubilei F, Culasso F. Stroke, disability, and dementia: Results of a population survey. Stroke. 1997;28(3):531-536.

12. Lowery K, Ballard C, Rodgers H, et al. Cognitive decline in a prospectively studied group of stroke survivors, with a particular emphasis on the $>75$ 's. Age Ageing. 2002;31 Suppl:324-327.

13. Pohjasvaara T, Erkinjuntti T, Ylikoski R, Hietanen M, Vataja R, Kaste M. Clinical determinants of poststroke dementia. Stroke. 1998;29(1):75-81.

14. Patel MD, Coshall C, Rudd AG, Wolfe CD. Cognitive impairment after stroke: Clinical determinants and its associations with long-term stroke outcomes. J Am Geriatr Soc. 2002;50(4):700-706.

15. Folstein MF, Folstein SE, McHugh PR. "Mini-mental state". A practical method for grading the cognitive state of patients for the clinician. J Psychiatr Res. 1975;12(3):189-198.

16. Schatz P, Hughes LJ, Chute DL. Underutilization of neuropsychology in traumatic brain injury rehabilitation: Is managed care to blame? NeuroRehabilitation. 2001;16(4):281-287.

17. Laukka EJ, Jones S, Fratiglioni L, Backman L. Cognitive functioning in preclinical vascular dementia: A 6-year follow-up. Stroke. 2004;35(8): 1805-1809.

18. Madureira S, Guerreiro M, Ferro JM. Dementia and cognitive impairment three months after stroke. Eur J Neurol. 2001;8(6):621-627.

19. Bonifer N, Anderson KM. Application of constraint-induced movement therapy for an individual with severe chronic upper-extremity hemiplegia. Phys Ther. 2003;83(4):384-398.

20. Bonifer NM, Anderson KM, Arciniegas DB. Constraint-induced movement therapy after stroke: Efficacy for patients with minimal upper-extremity motor ability. Arch Phys Med Rehabil. 2005; 86(9):1867-1873.

21. Bonifer NM, Anderson KM, Arciniegas DB. Constraint-induced therapy for moderate chronic upper extremity impairment after stroke. Brain Inj. 2005;19(5):323-330.

22. Fugl-Meyer AR, Jaasko L, Leyman I, Olsson S, Steglind S. The poststroke hemiplegic patient. 1. A method for evaluation of physical performance. Scand J Rehabil Med. 1975;7(1):13-31.

23. Crum RM, Anthony JC, Bassett SS, Folstein MF. Population-based norms for the Mini-Mental State Examination by age and educational level. JAMA. 1993;269(18):2386-2391.

24. Meyer JS, Xu G, Thornby J, Chowdhury MH, Quach M. Is mild cognitive impairment prodromal for vascular dementia like Alzheimer's disease? Stroke. 2002;33(8):1981-1985.

25. Bour A, Rasquin S, Boreas A, Limburg M, Verhey F. How predictive is the MMSE for cognitive performance after stroke? J Neurol. 2010;257(4):630-637.

26. Cumming TB, Blomstrand C, Bernhardt J, Linden T. The NIH stroke scale can establish cognitive function after stroke. Cerebrovasc Dis. 2010;30(1):7-14.

27. Te Winkel-Witlox AC, Post MW, Visser-Meily JM, Lindeman E. Efficient screening of cognitive dysfunction in stroke patients: Comparison between the CAMCOG and the R-CAMCOG, Mini Mental State Examination and Functional Independence Measure-cognition score. Disabil Rehabil. 2008;30(18):1386-1391.

28. Kelly-Hayes M, Robertson JT, Broderick JP, et al. The American Heart Association Stroke Outcome Classification. Stroke. 1998;29(6): 1274-1280. 
29. Popovic IM, Seric V, Demarin V. Mild cognitive impairment in symptomatic and asymptomatic cerebrovascular disease. J Neurol Sci. 2007;257(1-2):185-1893.

30. Srikanth V, Thrift AG, Fryer JL, et al. The validity of brief screening cognitive instruments in the diagnosis of cognitive impairment and dementia after first-ever stroke. Int Psychogeriatr. 2006;18(2):295-305.

31. Dong Y, Sharma VK, Chan BP, et al. The Montreal Cognitive Assessment (MoCA) is superior to the Mini-Mental State Examination (MMSE) for the detection of vascular cognitive impairment after acute stroke. J Neurol Sci. 2010;299(1-2):15-18.

32. Pendlebury ST, Cuthbertson FC, Welch SJ, Mehta Z, Rothwell PM. Underestimation of cognitive impairment by Mini-Mental State Examination versus the Montreal Cognitive Assessment in patients with transient ischemic attack and stroke: A population-based study. Stroke. 2010;41(6):1290-1293.

33. Srikanth VK, Quinn SJ, Donnan GA, Saling MM, Thrift AG. Long-term cognitive transitions, rates of cognitive change, and predictors of incident dementia in a population-based first-ever stroke cohort. Stroke. 2006;37(10):2479-2483.

34. Appelros P, Andersson AG. Changes in Mini Mental State Examination score after stroke: Lacunar infarction predicts cognitive decline. Eur J Neurol. 2006;13(5):491-495.

35. Ishikawa H, Meguro K, Ishii H, Tanaka N, Yamaguchi S. Silent infarction or white matter hyperintensity and impaired attention task scores in a nondemented population: The Osaki-Tajiri project. J Stroke Cerebrovasc Dis. October 22, 2010. [Epub ahead of print].

36. Podgorska A, Hier DB, Pytlewski A, Czlonkowska A. Leukoaraiosis and stroke outcome. J Stroke Cerebrovasc Dis. 2002;11(6):336-340.

37. Blass JP, Ratan RR. "Silent" strokes and dementia. N Engl J Med. 2003;348(13):1277-1278.

38. Decarli C. Vascular factors in dementia: An overview. J Neurol Sci. 2004;226(1-2):19-23.

39. Weller RO, Yow HY, Preston SD, Mazanti I, Nicoll JA. Cerebrovascular disease is a major factor in the failure of elimination of Abeta from the aging human brain: Implications for therapy of Alzheimer's disease. Ann N Y Acad Sci. 2002;977:162-168.

40. Zlokovic BV, Deane R, Sallstrom J, Chow N, Miano JM. Neurovascular pathways and Alzheimer amyloid beta-peptide. Brain Pathol. 2005; 15(1):78-83.

41. Mok V, Leung EY, Chu W, et al. Pittsburgh compound B binding in poststroke dementia. J Neurol Sci. 2010;290(1-2):135-137.

42. Bour AM, Rasquin SM, Baars L, et al. The effect of the APOE-epsilon4 allele and ACE-I/D polymorphism on cognition during a two-year follow-up in first-ever stroke patients. Dement Geriatr Cogn Disord. 2010;29(6):534-542.

43. Khedr EM, Hamed SA, El-Shereef HK, et al. Cognitive impairment after cerebrovascular stroke: Relationship to vascular risk factors. Neuropsychiatr Dis Treat. 2009;5:103-116.

44. Rothenburg LS, Herrmann N, Swardfager W, et al. The relationship between inflammatory markers and post stroke cognitive impairment. J Geriatr Psychiatry Neurol. 2010;23(3):199-205.
45. Newman GC, Bang H, Hussain SI, Toole JF. Association of diabetes, homocysteine, and HDL with cognition and disability after stroke. Neurology. 2007;69(22):2054-2062.

46. Raji MA, Reyes-Ortiz CA, Kuo YF, Markides KS, Ottenbacher KJ. Depressive symptoms and cognitive change in older Mexican Americans. J Geriatr Psychiatry Neurol. 2007;20(3):145-152.

47. Honig LS, Tang MX, Albert S, et al. Stroke and the risk of Alzheimer disease. Arch Neurol. 2003;60(12):1707-1712.

48. Kalaria RN. The role of cerebral ischemia in Alzheimer's disease. Neurobiol Aging. 2000;21(2):321-330.

49. Vermeer SE, Prins ND, den Heijer T, Hofman A, Koudstaal PJ, Breteler MM. Silent brain infarcts and the risk of dementia and cognitive decline. $N$ Engl J Med. 2003;348(13):1215-1222.

50. Hurley BF. Age, gender, and muscular strength. J Gerontol A Biol Sci Med Sci. 1995;50 Spec No. 41-44.

51. Brott T, Adams HP Jr, Olinger CP, et al. Measurements of acute cerebral infarction: A clinical examination scale. Stroke. 1989;20(7):864-870.

52. Melkas S, Oksala NK, Jokinen H, et al. Poststroke dementia predicts poor survival in long-term follow-up: Influence of prestroke cognitive decline and previous stroke. J Neurol Neurosurg Psychiatry. 2009;80(8):865-870.

53. Oksala NK, Jokinen H, Melkas S, et al. Cognitive impairment predicts poststroke death in long-term follow-up. J Neurol Neurosurg Psychiatry. 2009;80(11):1230-1235.

54. Appelros P, Nydevik I, Terent A. Living setting and utilisation of ADL assistance one year after a stroke with special reference to gender differences. Disabil Rehabil. 2006;28(1):43-49.

55. Malouf R, Birks J. Donepezil for vascular cognitive impairment. Cochrane Database Syst Rev. 2004(1):CD004395.

56. Moretti R, Torre P, Antonello RM, et al. Rivastigmine superior to aspirin plus nimodipine in subcortical vascular dementia: An open, 16-month, comparative study. Int J Clin Pract. 2004;58(4):346-353.

57. Erkinjuntti T, Roman G, Gauthier S. Treatment of vascular dementia evidence from clinical trials with cholinesterase inhibitors. J Neurol Sci. 2004;226(1-2):63-66.

58. Small G, Erkinjuntti T, Kurz A, Lilienfeld S. Galantamine in the treatment of cognitive decline in patients with vascular dementia or Alzheimer's disease with cerebrovascular disease. CNS Drugs. 2003;17(12):905-914.

59. Wilkinson D, Doody R, Helme R, et al. Donepezil in vascular dementia: A randomized, placebo-controlled study. Neurology. 2003;61(4):479-486.

60. Wilcock G, Mobius HJ, Stoffler A. A double-blind, placebo-controlled multicentre study of memantine in mild to moderate vascular dementia (MMM500). Int Clin Psychopharmacol. 2002;17(6):297-305.

61. Orgogozo JM, Rigaud AS, Stoffler A, Mobius HJ, Forette F. Efficacy and safety of memantine in patients with mild to moderate vascular dementia: A randomized, placebo-controlled trial (MMM 300). Stroke. 2002;33(7):1834-1839.
Neuropsychiatric Disease and Treatment

\section{Publish your work in this journal}

Neuropsychiatric Disease and Treatment is an international, peerreviewed journal of clinical therapeutics and pharmacology focusing on concise rapid reporting of clinical or pre-clinical studies on a range of neuropsychiatric and neurological disorders. This journal is indexed on PubMed Central, the 'PsycINFO' database and CAS, and is the official

\section{Dovepress}

journal of The International Neuropsychiatric Association (INA). The manuscript management system is completely online and includes a very quick and fair peer-review system, which is all easy to use. Visit http://www.dovepress.com/testimonials.php to read real quotes from published authors. 